\title{
Correction to: One-pot synthesis and characterization of in-house engineered silver nanoparticles from Flacourtia jangomas fruit extract with effective antibacterial profiles
}

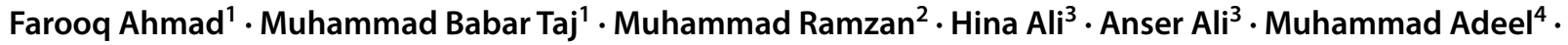 \\ Hafiz M. N. Iqbal ${ }^{5}$ (D) Muhammad Imran ${ }^{1}$
}

Published online: 4 January 2021

(c) Islamic Azad University 2021

\section{Correction to: \\ Journal of Nanostructure in Chemistry (2020) \\ https://doi.org/10.1007/s40097-020-00354-w}

We the listed author(s) are pointing out some unintentionally leftover irregularities, typos and mistakes which should be corrected to justify and validate the antibacterial evaluation.

Hereby, we bring to your attention the following irregularities, typos and mistakes in [1].

\begin{abstract}
Nanobiotechnology is an emerging field among researchers. Currently the development of plant-mediated biogenic
\end{abstract}

Farooq Ahmad and Muhammad Babar Taj authors have equal contribution and consider as the first author.

The original article can be found online at https://doi.org/10.1007/ s40097-020-00354-w.

Hafiz M. N. Iqbal

hafiz.iqbal@tec.mx

$\triangle$ Muhammad Imran

muhammad.imran@iub.edu.pk

1 Department of Chemistry, Baghdad-Ul-Jadeed Campus, The Islamia University of Bahawalpur, Bahawalpur 63100, Pakistan

2 Department of Physics, Baghdad-Ul-Jadeed Campus, The Islamia University of Bahawalpur, Bahawalpur 63100, Pakistan

3 Department of Zoology, Mirpur University of Science and Technology (MUST), Mirpur 10250, AJK, Pakistan

4 Metal Matrix Composite Laboratory, School of Chemistry and Chemical Engineering, Shanghai Jiao Tong University, Shanghai 200240, China

5 Tecnologico de Monterrey, School of Engineering and Sciences, 64849 Monterrey, Mexico nanoparticles is gaining much attention. It is notable that the biogenic nanoparticles particularly silver nanoparticles are therapeutically more effective than its chemically synthesized form. Thus, here in, the development of silver nanoparticles (Fj-AgNPs) using aqueous extract of ripe fruit of Flacourtia jangomas as a reducing agent is investigated. The developed Fj-AgNPs were additionally compared with ammine modified silver nanoparticles (Am-AgNPs), developed through chemical route using the reported approach and confirm the phyto-reduction of $\mathrm{Ag}^{+}$to $\mathrm{Ag}^{0}$.UV-visible spectrum has exhibited a peak at $418 \mathrm{~nm}$ and FTIR peak profile (at 1587.6, 1386.4, and $1076 \mathrm{~cm}^{-1}$ with corresponding compounds) in addition to the diffraction peak at $38^{\circ}$, $44^{\circ}, 64^{\circ}, 78^{\circ}$ in PXRD spectrum confirmed the synthesis of Fj-AgNPs with $8.29 \mathrm{~nm}$ average crystallite size. Enhanced anti-bacterial effect is observed in the case of Fj-AgNPs as compared to Am-AgNps. These results refer to the idea that the use of the aqueous extract of ripe fruit of Flacourtia jangomas could be a good replacement of EDTA in the synthesis of silver nanoparticles.

In methodology, under the section "Strains and culture conditions", the bacterial strain, i.e., Escherichia coli (ATTC 8723) must read as: Escherichia coli (ATTC 8739). The strain "S. enterica" must read as: Salmonella enterica.

In methodology, the section "Antibacterial activity of FjAgNps and Am-AgNps" must read as:

Antibacterial activity of Fj-AgNPs and Am-AgNPs was determined by using well diffusion method same as used previously [2]. Fresh bacterial cultures were prepared for each experiment. Then, $70 \mu \mathrm{L}$ bacterial suspension $(0.060$ OD at 600 wavelengths) was spread on agar plate homogeneously. After air dry $(10 \mathrm{~min})$ at room temperature (RT), 5 wells ( $6 \mathrm{~mm}$ diameter) were made using sterilized steel borer on each plate which were loaded with $50 \mu \mathrm{L}$ of test 
compounds having $0 \mathrm{mg} / \mathrm{mL}$ (control), $0.75 \mathrm{mg} / \mathrm{mL}, 1.5 \mathrm{mg} /$ $\mathrm{mL}, 3 \mathrm{mg} / \mathrm{mL}$ and $6 \mathrm{mg} / \mathrm{mL}$ concentrations, respectively. Later, all plates were placed in an incubator at $37{ }^{\circ} \mathrm{C}$ for $24 \mathrm{~h}$. Finally, plates were photographed, and zones of inhibition were measured in $\mathrm{cm}$. The same protocol has been employed to access the anti-bacterial effect of dimethyl sulfoxide (DMSO), plant extract, ethylenediaminetetraacetic acid (EDTA), and ciprofloxacin for the better understanding of anti-bacterial nature of synthesized nanomaterials.

In results and discussion, the section "Antibacterial potential of Fj-AgNPs in comparison to Am-AgNPs" must read as:

To discuss the comparison between the bacterial inhibition potential of Fj-AgNps and Am-AgNps, the antibacterial activities of plant extract $(6 \mathrm{mg} / \mathrm{mL})$, EDTA $(6 \mathrm{mg} / \mathrm{mL})$, $\mathrm{Fj}-\mathrm{AgNps}$ and $\mathrm{Am}-\mathrm{AgNps}$ were evaluated against five pathogenic bacteria namely Escherichia coli (E. coli), Bacillus cereus (B. cereus), Staphylococcus pyogen (S. pyogen), Salmonella enterica (S. enterica) and Staphylococcus aureus ( $S$. aureus) using well diffusion method. Later, zones of inhibition were measured as a representative of bacterial susceptibility at different concentrations of a test compound [3].

Both Fj-AgNPs and Am-AgNPs showed concentration dependent and bacterial species dependent killing effect as shown in Fig. 1. The anti-bacterial results of DMSO, plant extract, EDTA, and ciprofloxacin have been given in Fig. 2. The antibacterial effect of Fj-AgNPs increases more as compared to Am-AgNps upon increasing the dose concentration, except S. pyogens as shown in Fig. 1. Although Am-AgNPs posses EDTA as a functionalized character which considered tremendous anti-bacterial agent. The anti-bacterial capability of the Am-AgNPs ought to be higher than biogenic silver nanoparticles. But in this comparative study the lesser efficiency of Am-AgNPs stands for the lesser ammine functionalization on the nanoparticles surface. This phase is verifiable in the FT-IR spectrum where the absorption peak strength of $\mathrm{N}-\mathrm{H}$ seems suppressed owing to very less functionalization.

The Fj-AgNPs tested concentrations, $0.75 \mathrm{mg} / \mathrm{mL}$, $1.5 \mathrm{mg} / \mathrm{mL}, 3 \mathrm{mg} / \mathrm{mL}$ and $6 \mathrm{mg} / \mathrm{mL}$ showed $0 \mathrm{~cm}$, $0.0266 \mathrm{~cm}, 0.1781 \mathrm{~cm}, 0.275 \mathrm{~cm}$ zones of inhibition against E. coli, $0.0172 \mathrm{~cm}, 0.0703 \mathrm{~cm}, 0.1438 \mathrm{~cm}, 0.2438 \mathrm{~cm}$ zones of inhibition against B. cereus, $0.0281 \mathrm{~cm}, 0.0625 \mathrm{~cm}$, $0.1156 \mathrm{~cm}, 0.2188 \mathrm{~cm}$ zones of the inhibition against $S$. pyogen, $0.1688 \mathrm{~cm}, 0.2656 \mathrm{~cm}, 0.3438 \mathrm{~cm}, 0.4813 \mathrm{~cm}$ zones of inhibition against $S$. enterica and $0.0609 \mathrm{~cm}, 0.1219 \mathrm{~cm}$, $0.2031 \mathrm{~cm}, 0.2375 \mathrm{~cm}$ zones of inhibition against $S$. aureus, respectively at $24 \mathrm{~h}$ incubation. Furthermore, our results confirmed that all tested strains showed susceptibility against Fj-AgNPs with different level however the S. enterica
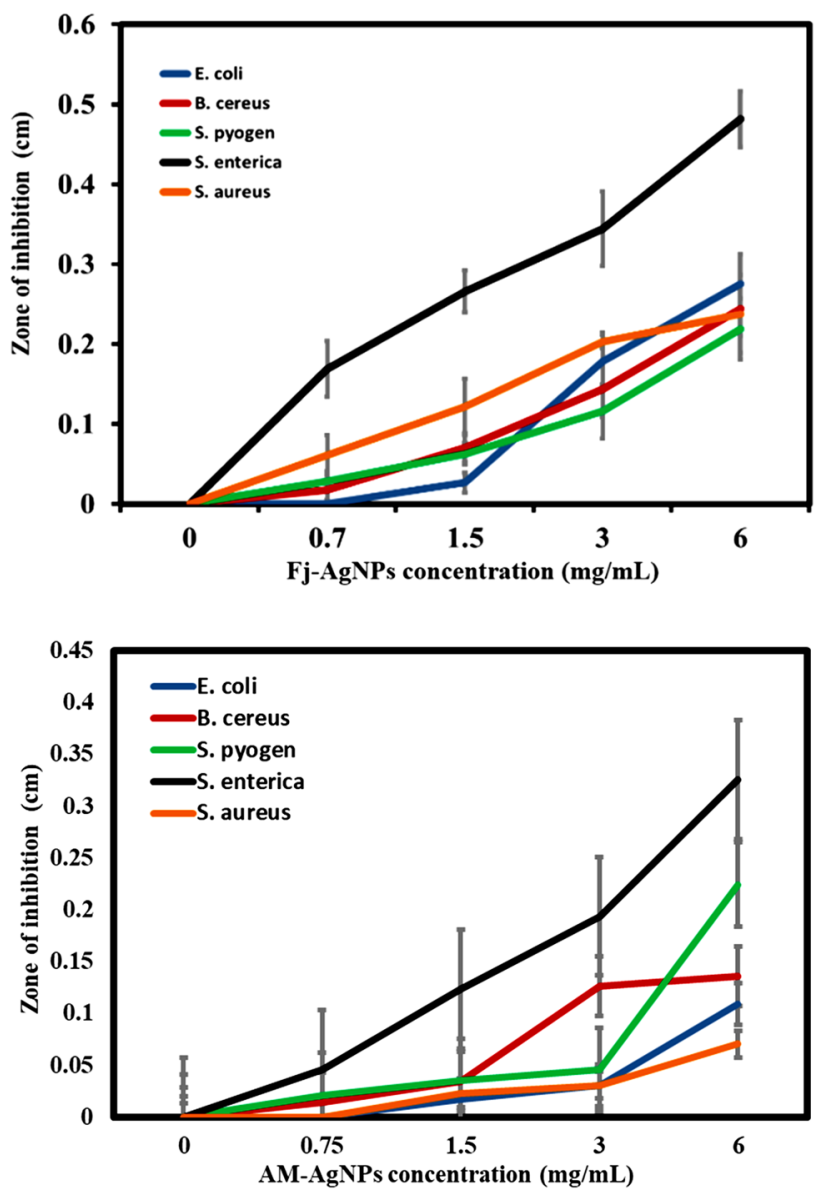

Fig. 1 Antibacterial susceptibility of Fj-AgNPs and Am-AgNPs against pathogenic bacteria

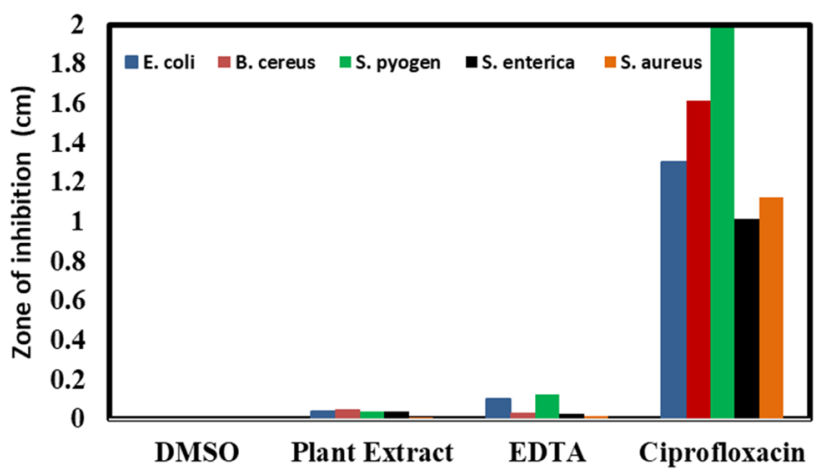

Fig. 2 Anti-bacterial potential of DMSO, plant extract, EDTA, and ciprofloxacin

showed the highest susceptibility at all tested concentrations (Fig. 1). The control having DMSO solvent $(0 \mathrm{mg} / \mathrm{mL}$ concentration of Ag-NPs) showed no zone of inhibition for all tested strains. It is expected that the silver ions block the functional operations of bacteria and interfere with the disulfide bonds which results in the structural deformation 
of protein. The principal achievement in bio-mediated over chemical fabrication is that it is eco-friendly, cost effective, non-toxic and feasible for commercial production without high temperature and high energy pressure and hazardous chemicals. Conclusively, the anti-bacterial potential of $\mathrm{Fj}^{-}$ AgNPs is in good comparison with the reported activities of biogenic AgNPs [4-6].

\section{Conclusion}

The synthesis of silver nanoparticles (Ag-NPs) in versatile ways has a deep impact on interdisciplinary science due to their various potential applications. With the present study, an extra-strength has been given for the synthesis using aqueous extract of ripe fruit of Flacourtia jangomas as it has good reducing power and potential against pathogenic microbes. The synthesized Fj-AgNps show comparatively good anti-microbial activity as equated to Am-AgNps. This enhanced antibacterial activity is due to the Flacourtia jangomas phytochemicals functionalization of silver nanoparticles during the synthesis of FJ-AgNPs.

The original article has been corrected.

\section{References}

1. Ahmad, F., Taj, M.B., Ramzan, M., Ali, H., Ali, A., Adeel, M., Imran, M., et al.: One-pot synthesis and characterization of in-house engineered silver nanoparticles from Flacourtia jangomas fruit extract with effective antibacterial profiles. J. Nanostruct. Chem. (2020). https://doi.org/10.1007/s40097-020-00354 $-\mathrm{W}$

2. Khurshid, H., Rafiq, M., Nazir, F., Ali, I., Ahmed, M., Akbar, B., Ali, A., et al.: Antimicrobial properties of hydrogen peroxide and potash alum alone and in combination against clinical bacterial isolates. Pure Appl. Biol. (PAB) 8(4), 2238-2247 (2019)

3. Han, D.W., Woo, Y.I., Lee, M.H., Lee, J.H., Lee, J., Park, J.C.: Invivo and in-vitro biocompatibility evaluations of silver nanoparticles with antimicrobial activity. J. Nanosci. Nanotechnol. 12(7), 5205-5209 (2012)

4. Sharma, V.K., Yngard, R.A., Lin, Y.: Silver nanoparticles: green synthesis and their antimicrobial activities. Adv. Colloid Interface. Sci. 145(1-2), 83-96 (2009)

5. Fatimah, I., Hidayat, H., Nugroho, B.H., Husein, S.: Ultrasoundassisted biosynthesis of silver and gold nanoparticles using Clitoria ternatea flower. S. Afr. J. Chem. Eng. 34, 97-106 (2020)

6. Rajathi, F.A.A., Parthiban, C., Kumar, V.G., Anantharaman, P.: Biosynthesis of antibacterial gold nanoparticles using brown alga, Stoechospermum marginatum (kützing). Spectrochim. Acta Part A Mol. Biomol. Spectrosc. 99, 166-173 (2012)

Publisher's Note Springer Nature remains neutral with regard to jurisdictional claims in published maps and institutional affiliations. 